\title{
A high positive correlation between the distance and temperature of the hottest nearby space objects
}

Bahram Kalhor ( $\sim$ kalhor_bahram@yahoo.com )

Islamic Azad University , Karaj https://orcid.org/0000-0002-6700-6269

Farzaneh Mehrparvar

Azad University

Behnam Kalhor

Karaj Branch, Azad University

\section{Research Article}

Keywords: distance, temperature, correlation, star, space

Posted Date: March 3rd, 2021

DOl: https://doi.org/10.21203/rs.3.rs-290084/v1

License: (c) (i) This work is licensed under a Creative Commons Attribution 4.0 International License.

Read Full License 


\title{
A high positive correlation between the distance and temperature of the hottest nearby space objects
}

Bahram Kalhor $^{1}$, Farzaneh Mehrparvar ${ }^{2}$, Behnam Kalhor ${ }^{3}$

\begin{abstract}
The paper uses the distance and temperature of 47,111 hottest nearby space objects including stars, quasars, white dwarf, and carbon stars. We have used SIMBAD Astronomical Database and obtained this information from 930,000 records. The range of temperature of the hottest objects is between 6158 and 99,575 K. Also, the distance of the objects is between 231.7375 and 1 (mas). We report the correlation between the distance and temperature of these hot objects at the temperature upper than $6632 \mathrm{~K}$ is equal to 0.135063 and will be increased to 0.32001 at temperatures upper than $9860 \mathrm{~K}$. Also, the correlation between the temperature and distance of objects hotter than $12,000 \mathrm{~K}$ is equal to 0.270218 .
\end{abstract}

Keywords: distance, temperature, correlation, star, space

\section{Introduction}

Recently, we have reported a positive correlation (0.914384) between the possibility of finding unexpected high Redshift ( $\mathrm{Z}$ greater than 0.001 ) and the average temperature of nearby objects in different categories [1]. By analyzing information of 199 high Redshift nearby objects we realized that most of them are quasar and white dwarf, and an average temperature of them is almost 9,771 K. The SIMBAD Astronomical Database told us "some of them showed up as stars in SDSS imaging data because they look like point sources, but they were followed up for spectra so that we can tell that they are actually quasars. The stars have Redshifts near zero and the quasars have Redshift values in the expected range". Hence, we decided to find the correlation between distance and temperature of hottest objects.

We obtained more than 930,000 records of hottest objects from the SIMBAD Astronomical Database and wrote a program for deleting repeated data. Finally, information of 47,111 hottest objects retrieved, including stars, quasars, white dwarf, and carbon stars. The distances of the objects are between 231.7375 and 1 (mas), and objects with redshift less than zero have been excluded.

Normally we do not expect to find a significant relationship between the distance of our nearby space objects and their temperature. The first results did not show a significant positive correlation of all objects with different temperatures, but after choosing objects with high temperatures, we found a significant positive correlation between temperature and distance of the hottest nearby space objects.

\footnotetext{
${ }^{1}$ Azad University, Karaj Branch. Email: bahram.kalhor@kiau.ac.ir

${ }^{2}$ Azad University, Karaj Branch, Department of Physics. Email: yekeh_Savar@yahoo.com

${ }^{3}$ Azad University, Karaj Branch, Department of Engineering. Email: b.kalhor@setareaval.ir

Corresponding author. Email: bahram.kalhor@kiau.ac.ir
} 
This paper is a statistical report, and we do not try to explain the reasons for this high correlation.

\section{Histogram}

In the SIMBAD Astronomical Database, the total number of the nearby space objects (distance less than 1 mas) with a temperature higher than $6158 \mathrm{~K}$ is equal to 47,111 . The SIMBAD Astronomical Database uses the Parallax method to obtain the distance of the space objects. Hence, we could expect precise distance of objects.

Table.1 shows the top hottest space objects and their distance. Most of them are quasars, white dwarf, and carbon stars.

Table.1: Top hottest nearby space objects

\begin{tabular}{|l|r|r|}
\hline \multicolumn{1}{|c|}{ Star Name } & Temperature & Parallax (mas) \\
\hline SDSS J092651.43+254859.0 & 99575 & 1.5027 \\
\hline SDSS J145545.58+041508.6 & 99575 & 1.658 \\
\hline 2QZ J133710.1-002644 & 95733.5 & 3.1521 \\
\hline SDSS J132858.19+590851.0 & 94514.5 & 6.6602 \\
\hline SDSS J200646.50-124410.9 & 93374.5 & 1.1089 \\
\hline NGC 4688 & 89520 & 1.7841 \\
\hline SDSS J211607.27+004503.2 & 88852.5 & 3.9088 \\
\hline SDSS J222203.33-003138.1 & 88434 & 1.4757 \\
\hline SDSS J100612.78+252833.6 & 87287.5 & 2.4609 \\
\hline SDSS J161613.09+252012.6 & 86556 & 1.5088 \\
\hline KUV 03459+0037 & 86359 & 2.5214 \\
\hline SH 2-216 & 84990 & 7.9404 \\
\hline SDSS J160236.07+381950.5 & 82904.5 & 1.1114 \\
\hline PN A66 7 & 82710 & 2.0204 \\
\hline SDSS J082153.01+190659.1 & 82213.6666666667 & 1.1727 \\
\hline HZ 34 & 82188.3333333333 & 1.6505 \\
\hline Ton 309 & 82168.8333333333 & 2.9982 \\
\hline PB 7489 & 78839.1428571429 & 1.907 \\
\hline GD 524 & 78750 & 21.1885 \\
\hline SDSS J101700.39+190110.1 & 78197.5 & 1.1778 \\
\hline SDSS J105555.23+484739.8 & 77370 & 1.2227 \\
\hline PG 1543+454 & 77293.3333333333 & 2.0611 \\
\hline HD 223816 & 76690 & 6.5857 \\
\hline WD 2121-076 & 76624.2 & 2.3702 \\
\hline SDSS J074632.00+415210.2 & 76569.4 & 1.3487 \\
\hline CD-45 5058 & 75858 & 1.7077 \\
\hline SDSS J101619.80-020258.2 & 75536 & 2.8029 \\
\hline LB 651 & 75062.5 & 1.7978 \\
\hline
\end{tabular}


Analyzing data of the hottest nearby objects shows that most of them are quasar, carbon star, or white dwarf. Hence, we will find the correlation between distance and temperature of them. Most of them have high redshift, and according to the SIMBAD Astronomical Database some of them showed up as stars in SDSS imaging data because they look like point sources

First, we present a histogram of the data. Regardless of the temperature of the space objects Fig.1 illustrates the histogram of them. The X-axis is the distance of the objects in the light year, and $\mathrm{Y}$-axis is the number of space objects. Earth is in an arm of the Milky Way. Hence, by increasing the distance, the number of space objects will be decreased, because of going to the free space out of the arm.

Fig.1: Histogram of 47,111 hottest nearby space objects.

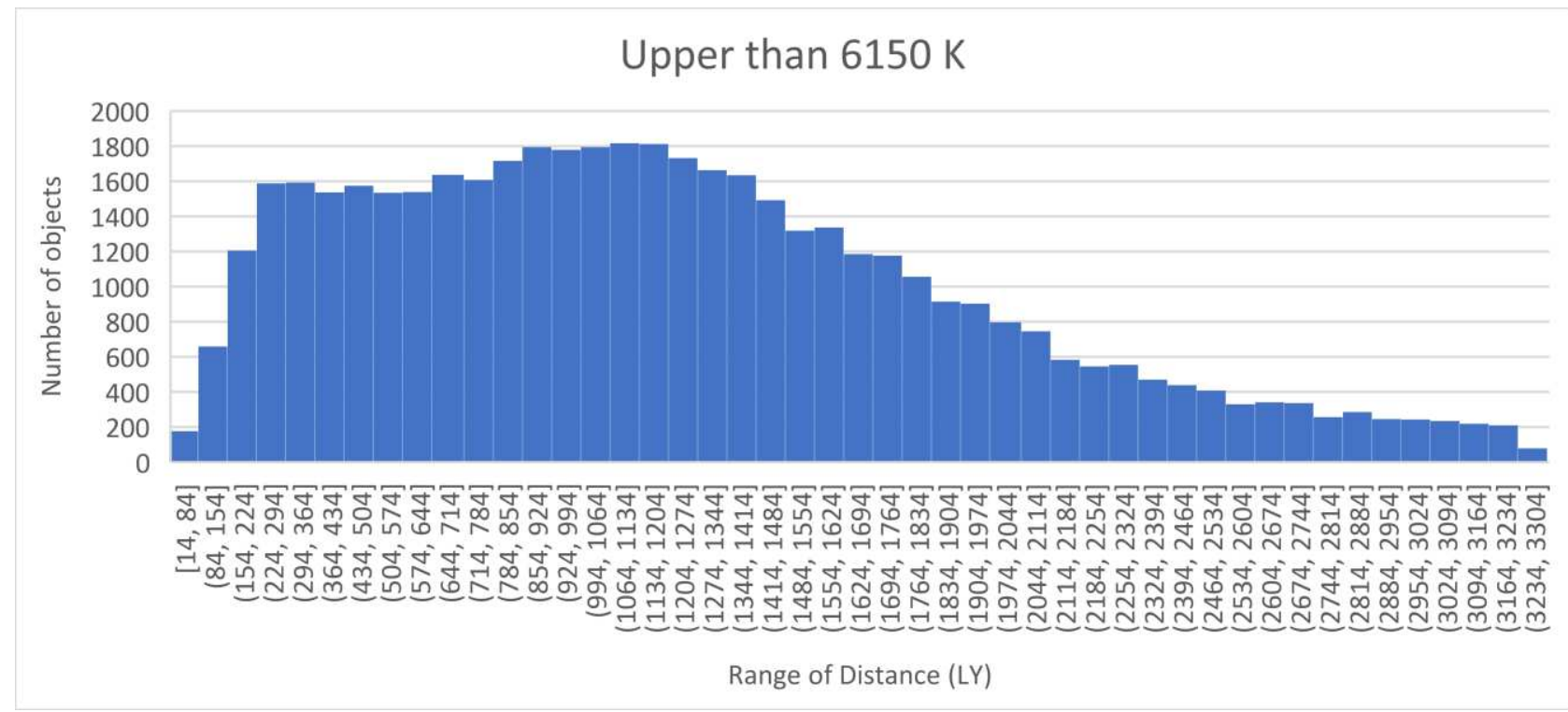

Fig.2: Histogram of 23,555 nearby space objects with temperature between 6155 and $6632 \mathrm{~K}$.

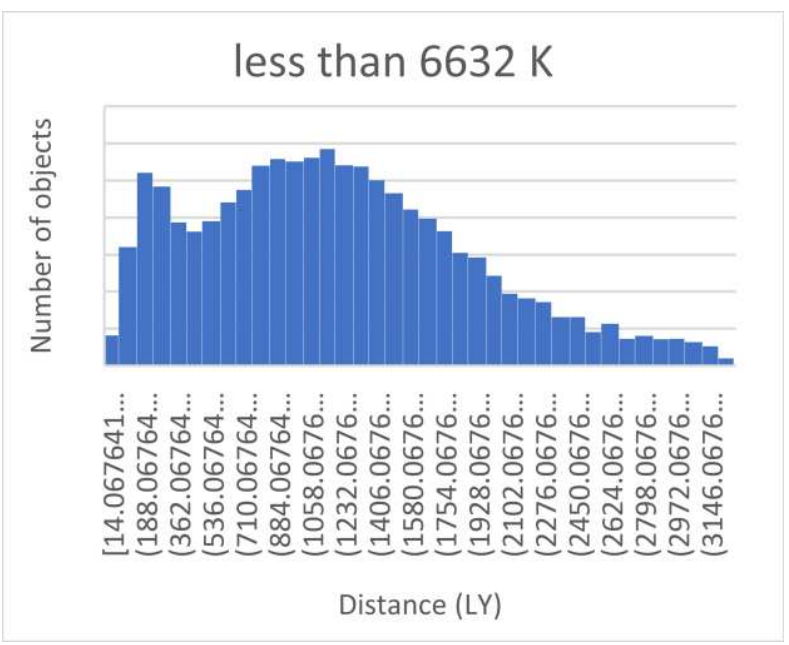

Fig.3: Histogram of 23,556 nearby space objects with temperature higher than $6632 \mathrm{~K}$.

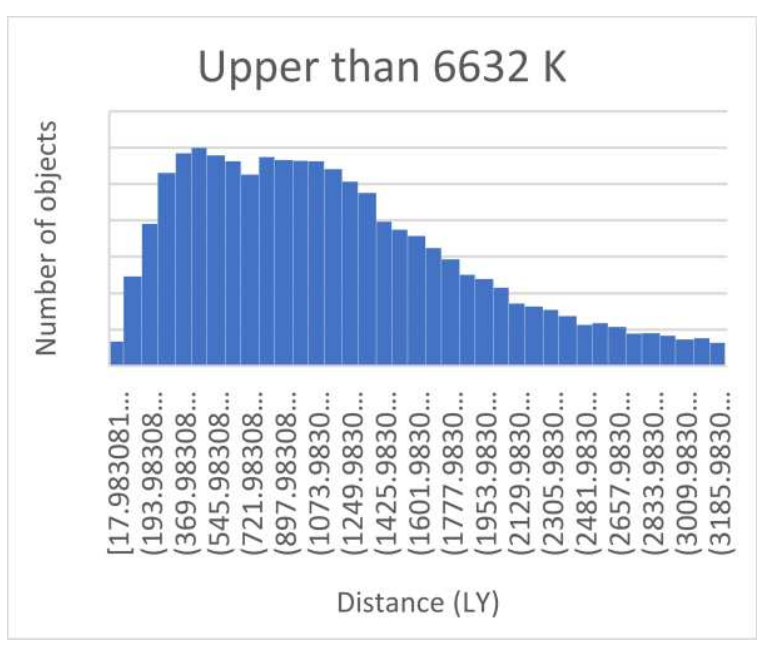


Fig.2 and Fig.3 illustrate the histogram of the nearby objects in two different categories of temperatures. Fig. 2 is a histogram of 23,555 objects with a temperature lower than $6632 \mathrm{~K}$, and Fig.3 illustrates a histogram of the 23,556 objects hotter than $6632 \mathrm{~K}$. The distribution of both categories is almost the same. Hence, we could not expect to find the different values of the correlation between distance and temperature of them.

\section{Results}

Firstly, we choose the biggest category, including all 47,111 space objects. The correlation between distance and temperature is equal to 0.076109063 , which is almost zero. Secondly, we obtain the correlation between distance and temperature of the 23,555 objects with a temperature lower than $6632 \mathrm{~K}$. The correlation between distance and temperature is equal to -0.03736 , which is less than zero. After choosing a new category of the objects, including the 23,556 objects hotter than $6632 \mathrm{~K}$, the correlation between distance and temperature will be obtained equal to 0.135063 , which is higher than the colder category.

We have obtained the correlation between distance and temperature for new categories, including hotter space objects. Table. 2 illustrates the relationship between the distance and temperature of the nearby objects. The correlation between distance and temperature of the space objects hotter than $9860 \mathrm{~K}$ is equal to 0.32001 .

Table.2: Correlation between distance and temperature

\begin{tabular}{|l|c|l|}
\hline \multicolumn{1}{|c|}{ Temperature (K) } & $\begin{array}{c}\text { Number of } \\
\text { objects }\end{array}$ & \multicolumn{1}{|c|}{$\begin{array}{c}\text { Correlation between } \\
\text { distance and temperature }\end{array}$} \\
\hline Greater than 6158 & 47,111 & 0.076109063 \\
\hline Between 6158 and 6632 & 23,555 & -0.03736 \\
\hline Greater than 6632 & 23,556 & 0.135063 \\
\hline Greater than 9860 & 8,924 & 0.32001 \\
\hline Greater than 12000 & 7,027 & 0.270218 \\
\hline Greater than 15000 & 4,882 & 0.194355 \\
\hline
\end{tabular}

\section{Acknowledgment}

This research has made use of the SIMBAD database, operated at CDS, Strasbourg, France 2000,A\&AS,143,9, "The SIMBAD astronomical database", Wenger et al.

\section{References}

1. Kalhor, Bahram; Mehrparvar, Farzaneh; kalhor, Behnam, "Impact of the temperature of stars on their Redshift." figshare. Preprint. https://doi.org/10.6084/m9.figshare.14009957.v1. (2021)

2. Kalhor, Bahram; Mehrparvar, Farzaneh; kalhor, Behnam. "Unexpected Redshift of nearby stars." figshare. Preprint. https://doi.org/10.6084/m9.figshare.13674298.v1. (2021). 
3. Riess, Adam G., et al. "Observational evidence from supernovae for an accelerating universe and a cosmological constant." The Astronomical Journal 116.3 (1998): 1009.

4. Wojtak, Radosław, Steen H. Hansen, and Jens Hjorth. "Gravitational Redshift of galaxies in clusters as predicted by general relativity." Nature 477.7366 (2011): 567-569.

5. Wolf, Peter, et al. "Atom gravimeters and gravitational Redshift." Nature 467.7311 (2010): E1-E1.

6. Brown, Zarah, et al. "A pole-to-pole pressure-temperature map of Saturn's thermosphere from Cassini Grand Finale data." Nature Astronomy 4.9 (2020): 872-879.

7. Kaaret, P., et al. "A disk-dominated and clumpy circumgalactic medium of the Milky Way seen in Xray emission." Nature Astronomy 4.11 (2020): 1072-1077.

8. Ruiz-Lara, Tomás, et al. "The recurrent impact of the Sagittarius dwarf on the star formation history of the Milky Way." Nature Astronomy 4.10 (2020): 965-973. 
Figures

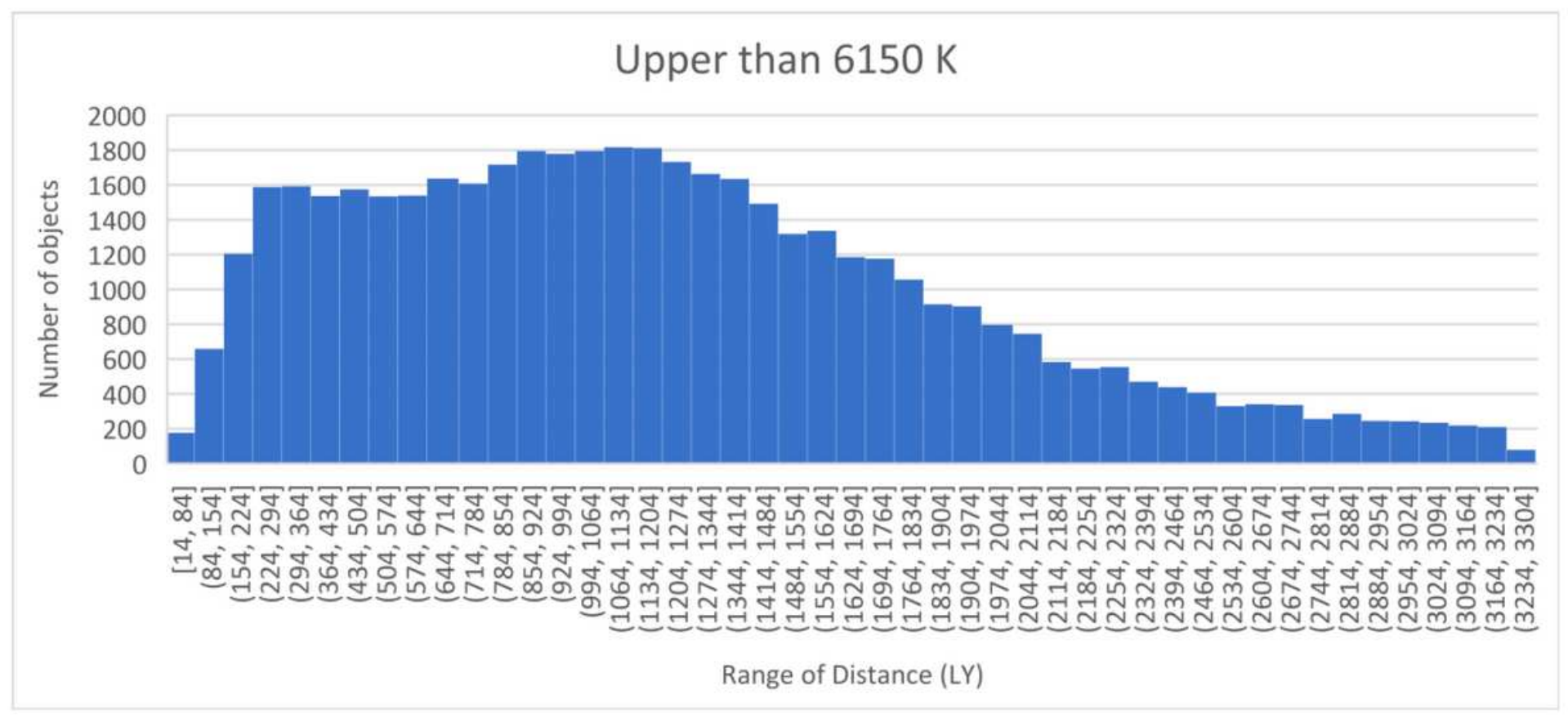

Figure 1

Histogram of 47,111 hottest nearby space objects. 


\section{less than $6632 \mathrm{~K}$}
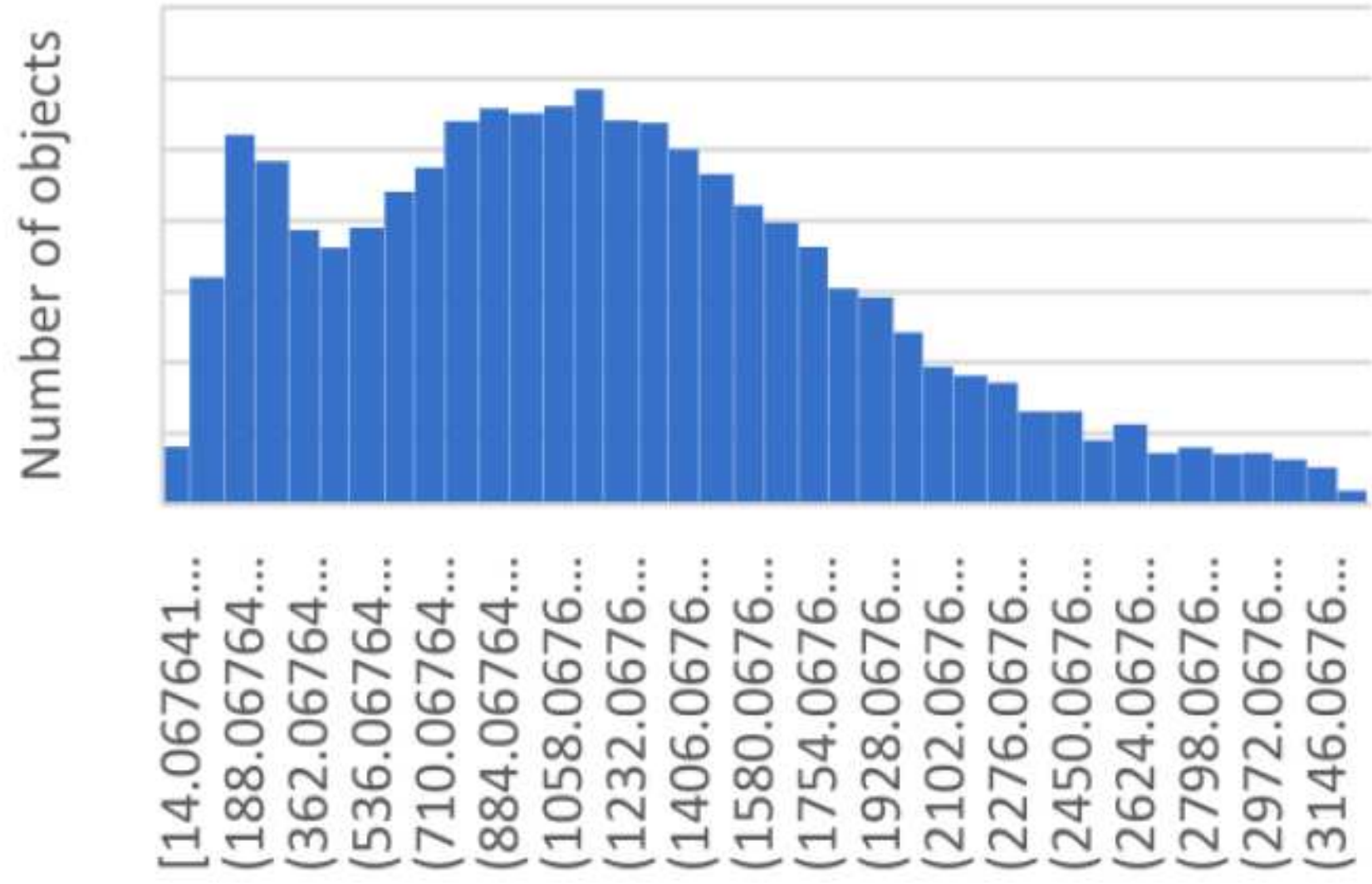

\section{Distance (LY)}

Figure 2

Histogram of 23,555 nearby space objects with temperature between 6155 and $6632 \mathrm{~K}$. 


\section{Upper than $6632 \mathrm{~K}$}

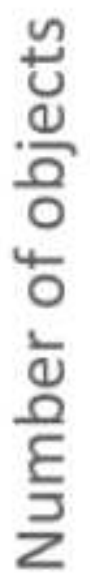

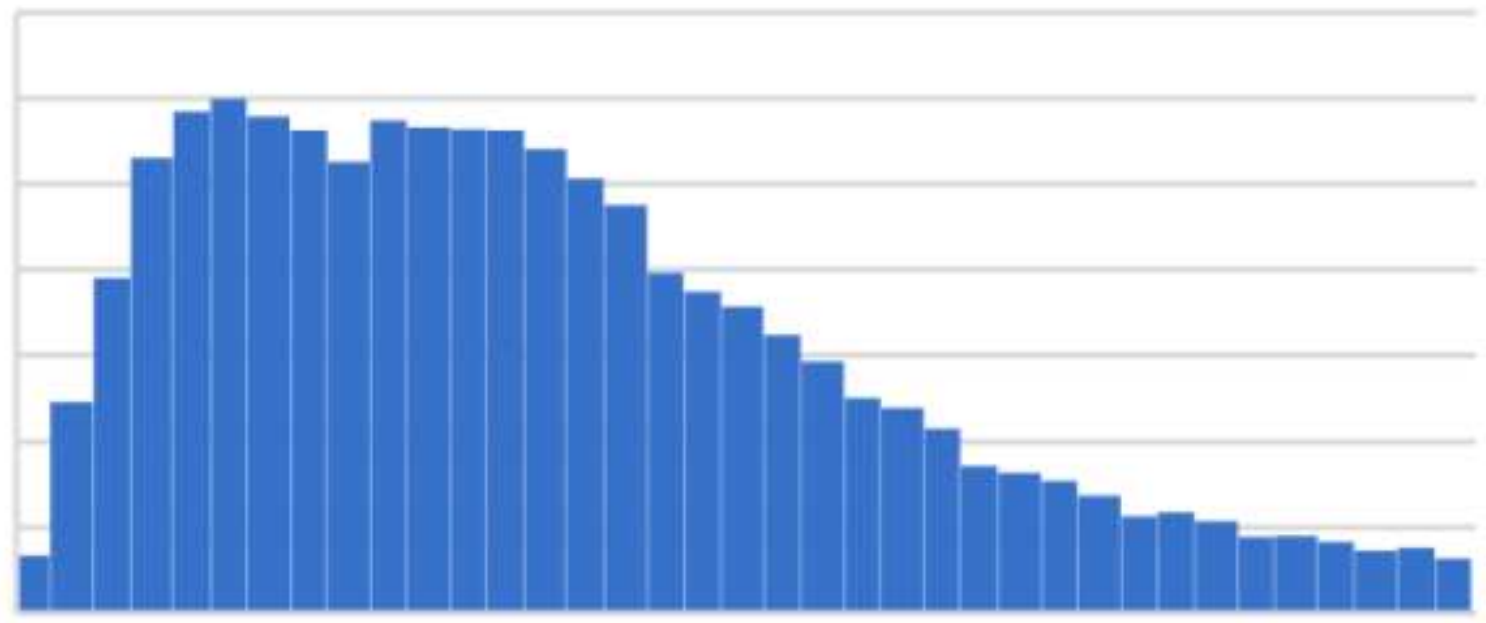

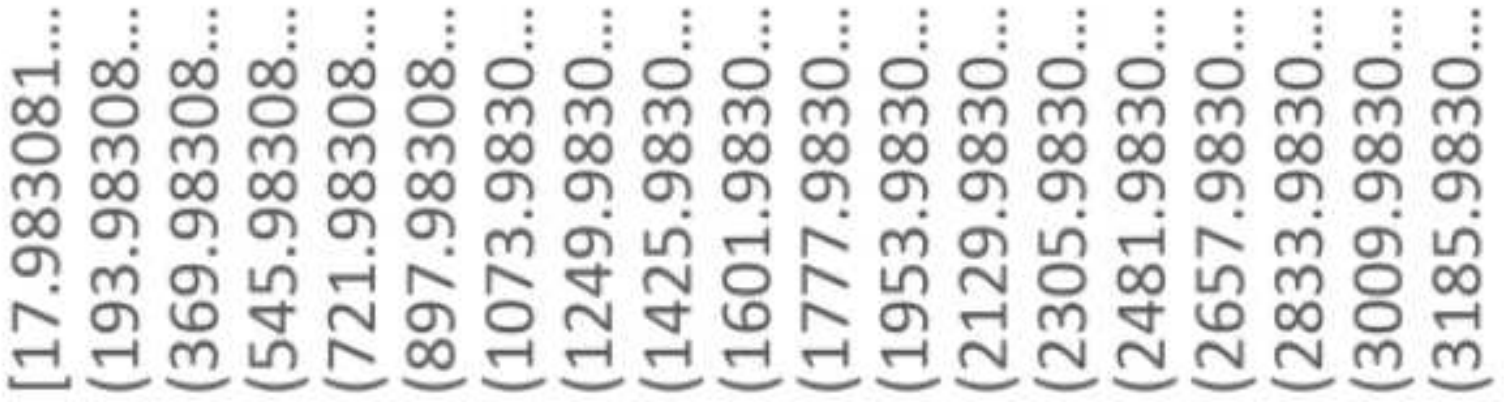

Distance (LY)

Figure 3

Histogram of 23,556 nearby space objects with temperature higher than $6632 \mathrm{~K}$. 